University of Wollongong

Research Online

Faculty of Engineering - Papers (Archive)

Faculty of Engineering and Information

Sciences

2002

\title{
Enhancement of the critical current density and flux pinning of MgB2 superconductor by nanoparticle SiC doping
}

\author{
S X. Dou \\ University of Wollongong, shi@uow.edu.au \\ Saeid Soltanian \\ University of Wollongong, saeid@uow.edu.au \\ J. Horvat \\ University of Wollongong, jhorvat@uow.edu.au \\ Xiaolin Wang \\ University of Wollongong, xiaolin@uow.edu.au
}

S. H. Zhou

University of Wollongong, sihai@uow.edu.au

See next page for additional authors

Follow this and additional works at: https://ro.uow.edu.au/engpapers

Part of the Engineering Commons

https://ro.uow.edu.au/engpapers/93

\section{Recommended Citation}

Dou, S X.; Soltanian, Saeid; Horvat, J.; Wang, Xiaolin; Zhou, S. H.; Ionescu, M.; Liu, Hua-Kun; Munroe, P.; and Tomsic, M.: Enhancement of the critical current density and flux pinning of MgB2 superconductor by nanoparticle SiC doping 2002.

https://ro.uow.edu.au/engpapers/93

Research Online is the open access institutional repository for the University of Wollongong. For further information contact the UOW Library: research-pubs@uow.edu.au 


\section{Authors}

S X. Dou, Saeid Soltanian, J. Horvat, Xiaolin Wang, S. H. Zhou, M. Ionescu, Hua-Kun Liu, P. Munroe, and M. Tomsic 


\title{
Enhancement of the critical current density and flux pinning of $\mathrm{MgB}_{2}$ superconductor by nanoparticle SiC doping
}

\author{
S. X. Dou, ${ }^{\text {a) }}$ S. Soltanian, J. Horvat, X. L. Wang, S. H. Zhou, M. Ionescu, and H. K. Liu \\ Institute for Superconducting and Electronic Materials, University of Wollongong, Northfields Avenue, \\ Wollongong, NSW 2522, Australia \\ P. Munroe \\ Electron Microscopy Unit, University of New South Wales, Sydney, NSW 2001, Australia \\ M. Tomsic \\ Hyper Tech Research Incorporated, 110 E. Canal Street, Troy, Ohio 45373
}

(Received 11 July 2002; accepted 5 September 2002)

\begin{abstract}
Doping of $\mathrm{MgB}_{2}$ by nano-SiC and its potential for the improvement of flux pinning were studied for $\mathrm{MgB}_{2-x}(\mathrm{SiC})_{x / 2}$ with $x=0,0.2$, and 0.3 and for 10 wt $\%$ nano-SiC-doped $\mathrm{MgB}_{2}$ samples. Cosubstitution of B by $\mathrm{Si}$ and $\mathrm{C}$ counterbalanced the effects of single-element doping, decreasing $T_{c}$ by only $1.5 \mathrm{~K}$, introducing intragrain pinning centers effective at high fields and temperatures, and significantly enhancing $J_{c}$ and $H_{\mathrm{irr}}$. Compared to the undoped sample, $J_{c}$ for the $10 \mathrm{wt} \%$ doped sample increased by a factor of 32 at $5 \mathrm{~K}$ and $8 \mathrm{~T}, 42$ at $20 \mathrm{~K}$ and $5 \mathrm{~T}$, and 14 at $30 \mathrm{~K}$ and $2 \mathrm{~T}$. At $20 \mathrm{~K}$ and $2 \mathrm{~T}$, the $J_{c}$ for the doped sample was $2.4 \times 10^{5} \mathrm{~A} / \mathrm{cm}^{2}$, which is comparable to $J_{c}$ values for the best $\mathrm{Ag} / \mathrm{Bi}-2223$ tapes. At $20 \mathrm{~K}$ and $4 \mathrm{~T}, J_{c}$ was twice as high as for the best $\mathrm{MgB}_{2}$ thin films and an order of magnitude higher than for the best $\mathrm{Fe} / \mathrm{MgB}_{2}$ tapes. The magnetic $J_{c}$ is consistent with the transport $J_{c}$ which remains at $20000 \mathrm{~A} / \mathrm{cm}^{2}$ even at $10 \mathrm{~T}$ and $5 \mathrm{~K}$ for the doped sample, an order of magnitude higher than the undoped one. Because of such high performance, it is anticipated that the future $\mathrm{MgB}_{2}$ conductors will be made using a formula of $\mathrm{MgB}_{x} \mathrm{Si}_{y} \mathrm{C}_{z}$ instead of pure $\mathrm{MgB}_{2}$. (C) 2002 American Institute of Physics. [DOI: 10.1063/1.1517398]
\end{abstract}

The critical current density $\left(J_{c}\right)$ in $\mathrm{MgB}_{2}$ has been a central topic of research since superconductivity in this compound was discovered. ${ }^{1}$ High $J_{c}$ values of $10^{5}$ to $10^{6} \mathrm{~A} / \mathrm{cm}^{2}$ have been reported for $\mathrm{MgB}_{2}$ by several groups. However, $J_{c}$ drops rapidly with increasing magnetic field due to its poor flux pinning. To take advantage of its high $T_{c}(39 \mathrm{~K})$, improvements in the irreversibility field $\left(H_{\text {irr }}\right)$ and $J_{c}(H)$ were achieved by oxygen alloying of $\mathrm{MgB}_{2}$ thin films ${ }^{2}$ and by proton irradiation of $\mathrm{MgB}_{2}$ powder. ${ }^{3}$ However, for practical applications, the pinning centers should be introduced by a simple process, such as chemical doping. Most of element substitution studies were aimed at raising $T_{c}$ and were thus limited to a low doping level. Improvement of flux pinning was also attempted, using doping by chemical compounds. Here, the results are largely limited to the addition of the compounds, rather than substitution of $\mathrm{Mg}$ or B by the compounds. Additives alone appear to be ineffective for the improvement of pinning at high temperatures. ${ }^{4-6}$ Recently, we found that chemical doping of nano-SiC into $\mathrm{MgB}_{2}$ can significantly enhance $J_{c}$ in high fields with only slight reductions in $T_{c}$ up to a doping level of $40 \%$ of B. ${ }^{7}$ This finding clearly demonstrated that cosubstitution of $\mathrm{SiC}$ for $\mathrm{B}$ in $\mathrm{MgB}_{2}$ induced intragrain defects and a high density of nanoinclusions as effective pinning centers. However, the processing conditions used were far from optimized and the sample density was only $50 \%$ of the theoretical value. Therefore, it was not possible to assess the full potential of nano-

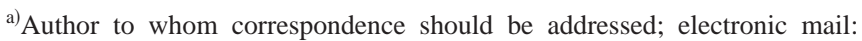
shi_dou@uow.edu.au
}

$\mathrm{SiC}$ doping for the improvement of $J_{c}$. In this work, we show that nanometer size $\mathrm{SiC}$-doped $\mathrm{MgB}_{2}$ gives the highest $J_{c}$ values in high magnetic fields at $20 \mathrm{~K}$ reported for any form of $\mathrm{MgB}_{2}$, including thin films.

$\mathrm{MgB}_{2}$ pellet samples were prepared by an in situ reaction method, which has been described in detail elsewhere. ${ }^{7}$ Powders of magnesium (99\% pure) and amorphous boron (99\% pure) were well mixed with $\mathrm{SiC}$ nanoparticle powder (size 10 to $100 \mathrm{~nm}$ ) with the atomic ratio of $\mathrm{MgB}_{2-x}(\mathrm{SiC})_{x / 2}$, where $x=0,0.2$, and 0.3 , for samples designated as 1 to 3 , respectively. A sample with $10 \mathrm{wt} \%$ of $\mathrm{SiC}$ addition to $\mathrm{MgB}_{2}$ was also made as sample 4 . Pellets $10 \mathrm{~mm}$ in diameter and $2 \mathrm{~mm}$ in thickness were made under uniaxial pressure, sealed in Fe tubes and then heated at temperatures of $700-900{ }^{\circ} \mathrm{C}$ for $1 \mathrm{~h}$ in flowing high-purity Ar. This was followed by furnace cooling to room temperature. The same powders used in samples 1 and 4 were made into wires using the powder-in-tube method. ${ }^{10}$ These are designated as samples 5 and 6 , respectively.

The magnetization of samples was measured over a temperature range of 5 to $30 \mathrm{~K}$ using a physical property measurement system (PPMS) (Quantum Design) in a timevarying magnetic field with sweep rate $50 \mathrm{Oe} / \mathrm{s}$ and amplitude up to $8.5 \mathrm{~T}$. All the samples were cut to the same size of $0.56 \times 2.17 \times 3.73 \mathrm{~mm}^{3}$ from as-sintered pellets. A magnetic $J_{c}$ was derived from the height of the magnetization loop $\Delta M$ using a Bean model: $J_{c}=20 \Delta M /[a(1$ $-a / 3 b)]$. Irreversibility field $\left(H_{\text {irr }}\right)$ was obtained from measuring the field-cooled and zero-field-cooled magnetic moments as a function of temperature for several values of the 


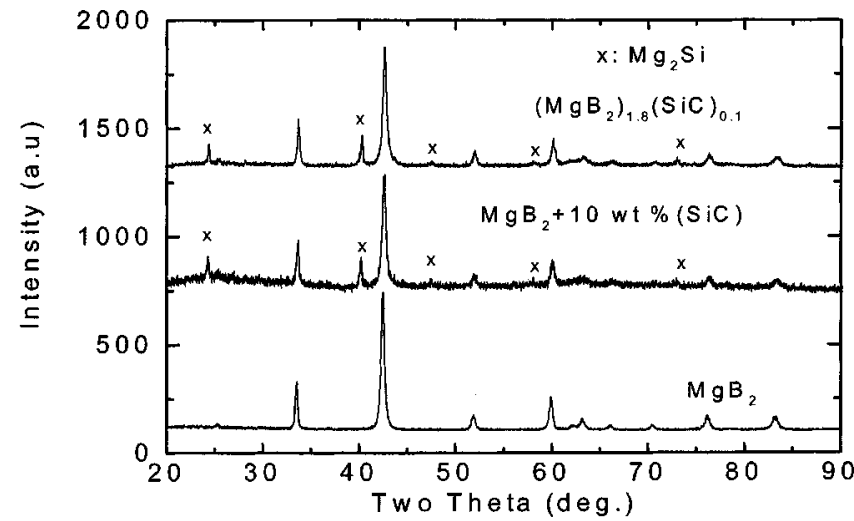

FIG. 1. XRD patterns for the nondoped and SiC-doped samples.

field. The critical temperature $\left(T_{c}\right)$ was obtained as the onset of the diamagnetic transition in the magnetic ac susceptibility measurements. The transport $J_{c}$ was measured with the four-probe method using a pulsed current source.

Figure 1 shows $\mathrm{x}$-ray diffraction (XRD) patterns for the $\mathrm{SiC}$ doped and nondoped samples. The XRD pattern for the nondoped sample (sample 1) reveals about $5 \% \mathrm{MgO}$, beside $\mathrm{MgB}_{2}$ as the main phase. Samples 2 and 3 consist of $\mathrm{MgB}_{2}$ as the main phase, with $\mathrm{Mg}_{2} \mathrm{Si}$ as the major impurity phase (crosses in Fig. 1). The estimated fraction of $\mathrm{Mg}_{2} \mathrm{Si}$ was $10 \%$. The energy dispersive spectroscopy (EDS) analysis results showed that the Mg:Si ratio was identical over the entire sample area, indicating a homogeneous phase distribution.

Figure 2 shows magnetic $J_{c}(H)$ curves for the SiCdoped $\mathrm{MgB}_{2}$ samples at $5 \mathrm{~K}, 20 \mathrm{~K}$, and $30 \mathrm{~K}$, for different doping levels. It is noted that all the $J_{c}(H)$ curves for doped samples show a crossover with the nondoped samples at higher fields. Although $\mathrm{SiC}$ doping caused a slight reduction of $J_{c}$ in low fields, it is much larger than for the nondoped samples in high fields for all the measured temperatures. Compared to the nondoped sample, $J_{c}$ for the $10 \mathrm{wt} \%$ doped sample increased by a factor of 32 at $5 \mathrm{~K}$ and $8 \mathrm{~T}, 23$ at $15 \mathrm{~K}$ and $6 \mathrm{~T}, 42$ at $20 \mathrm{~K}$ and $5 \mathrm{~T}$, and 14 at $30 \mathrm{~K}$ and $2 \mathrm{~T}$. This is the best $J_{c}(H)$ performance ever reported for $\mathrm{MgB}_{2}$ in any form. It is noted that the $J_{c}(H)$ curves for the nondoped sample showed a rapid drop in high fields and a plateau near $H_{\text {irr }}$. Earlier, we ascribed this phenomenon to the grains de-

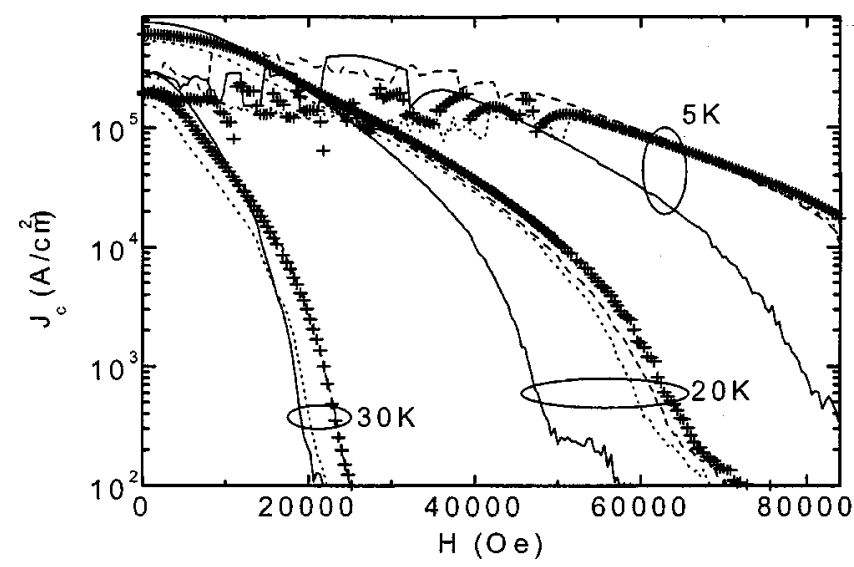

FIG. 2. The magnetic $J_{c}$ dependence at 5, 20, and $30 \mathrm{~K}$ for samples $1,2,3$, and 4 , shown by solid, dashed, and dotted lines, and crosses, respectively.

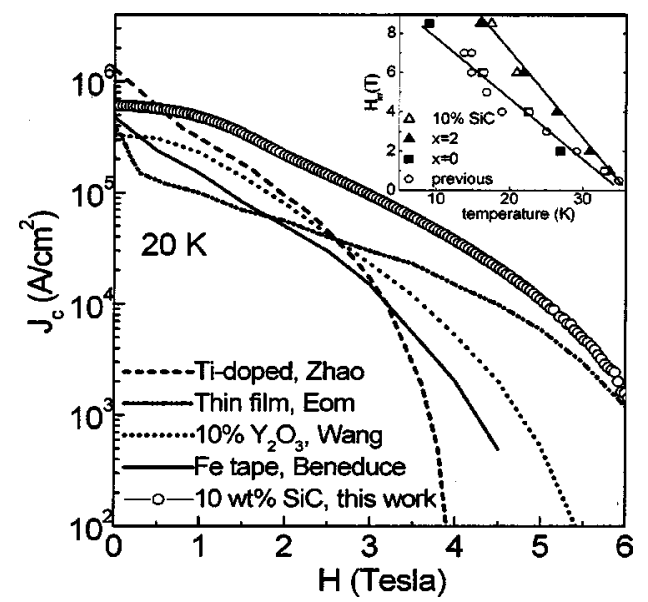

FIG. 3. A comparison of magnetic $J_{c}(H)$ at $20 \mathrm{~K}$ for the $10 \mathrm{wt} \% \mathrm{SiC}$-doped sample (sample 4) and for samples that were: Ti doped (see Ref. 4), $\mathrm{Y}_{2} \mathrm{O}_{3}$ (see Ref. 5) doped, thin film with strong pinning (see Ref. 2) and Fe/MgB tape (see Ref. 9). Inset: temperature dependence of the irreversibility field for SiC-doped $\mathrm{MgB}_{2}$ with different $\mathrm{SiC}$ content (triangles and squares) and for previously prepared nondoped $\mathrm{MgB}_{2}$ (round symbols).

coupling at higher fields, as a consequence of impurities at the grain boundaries. ${ }^{8}$ In contrast, none of the SiC-doped samples show this phenomenon, as either the substitutions or the induced nanoinclusions are incorporated into the grains.

Figure 3 shows a comparison of magnetic $J_{c}(H)$ for a 10 wt $\%$ SiC-doped sample at $20 \mathrm{~K}$ with data reported in literature. $J_{c}$ for this sample exhibits a better field performance and higher values of $J_{c}$ in high field than any other element doped samples ${ }^{4-6}$ or nondoped wires. ${ }^{9}$ Our SiC-doped $\mathrm{MgB}_{2}$ is even better than the thin-film $\mathrm{MgB}_{2}$ (Fig. 3), which had exhibited the strongest reported flux pinning and the highest $J_{c}$ in high fields to date. At $20 \mathrm{~K}$, the best $J_{c}$ for the $10 \mathrm{wt} \%$ SiC-doped sample was $10^{5} \mathrm{~A} / \mathrm{cm}^{2}$ at $3 \mathrm{~T}$, which exceeds the $J_{c}$ values of state-of-the-art $\mathrm{Ag} / \mathrm{Bi}-2223$ tapes. At $20 \mathrm{~K}$ and 4 $\mathrm{T}, J_{c}$ was $36000 \mathrm{~A} / \mathrm{cm}^{2}$, which is twice as high as for the best $\mathrm{MgB}_{2}$ thin films ${ }^{2}$ and an order of magnitude higher than for the state-of-the-art $\mathrm{Fe} / \mathrm{MgB}_{2}$ tapes. ${ }^{9}$

The temperature dependence of $H_{\text {irr }}$ for nano-SiC-doped $\mathrm{MgB}_{2}$, as well as for the pellets and tapes prepared previously (round symbols), is shown in the inset to Fig. 3. Apparently, $H_{\text {irr }}$ for $x=0$ overlaps with $H_{\text {irr }}$ for the previous samples, even though the latter had significantly smaller values of $J_{c}$. Doping with $\mathrm{SiC}$ significantly improved $H_{\mathrm{irr}}$. For example, $H_{\text {irr }}$ for the $\mathrm{SiC}$-doped samples reached $7.3 \mathrm{~T}$ at 20 $\mathrm{K}$, compared to $5.7 \mathrm{~T}$ for the nondoped one. This is consistent with improvement of the field dependence of $J_{c}$ with the doping. Because $H_{\text {irr }}$ for the nondoped control sample ( $x$ $=0$ ) is the same as for the previously prepared samples, the improvement of $J_{c}(H)$ definitely occurred because of the improvement of flux pinning by the doping and not because of improved sintering of $\mathrm{MgB}_{2}$.

Figure 4 shows the transport $J_{c}(H)$ values for the undoped and $10 \mathrm{wt} \% \mathrm{SiC}$-doped $\mathrm{MgB}_{2}$ wires (samples 5 and 6) at $5 \mathrm{~K}, 10 \mathrm{~K}$, and $20 \mathrm{~K}$. It is evident that the transport $J_{c}$ results for both the undoped and doped wires are in excellent agreement with the magnetic $J_{c}$. It is also clear that the enhancement in transport $J_{c}$ due to $\mathrm{SiC}$ doping is consistent with the magnetic $J_{c}$. The transport $I_{c}$ for the $10 \mathrm{wt} \% \mathrm{SiC}$ doped $\mathrm{MgB}_{2} / \mathrm{Fe}$ reached $660 \mathrm{~A}$ at $5 \mathrm{~K}$ and $4.5 \mathrm{~T}\left(J_{c}\right.$ o AlP license or copyright, see http://apl.aip.org/apl/copyright.jsp 


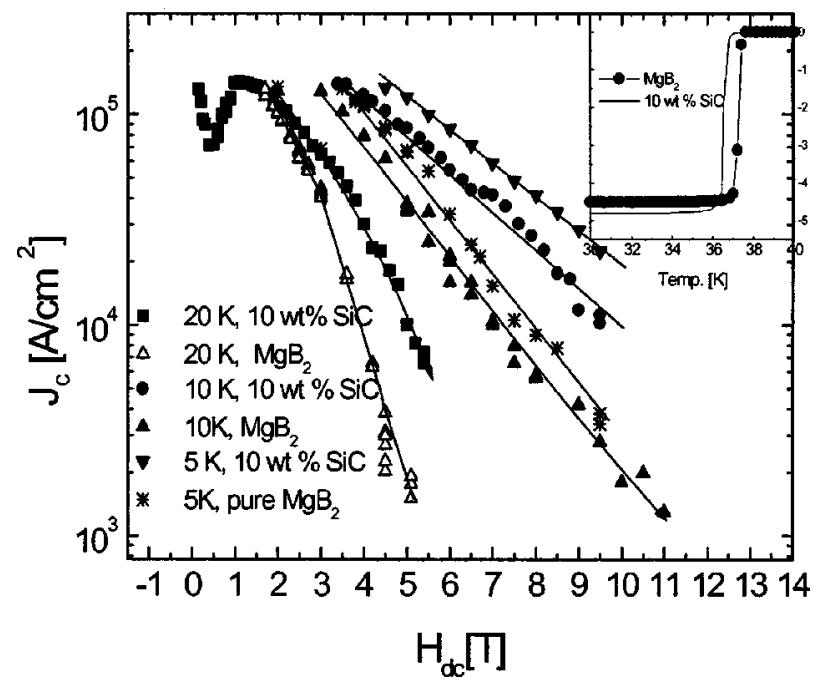

FIG. 4. Transport $J_{c}$ for $\mathrm{SiC}$ doped (sample 5) and undoped $\mathrm{MgB}_{2}$ (sample 6) wires (the lines only serve for guiding the eyes). Inset shows $T_{c}$ for these samples.

$\left.=133000 \mathrm{~A} / \mathrm{cm}^{2}\right)$ and $540 \mathrm{~A}$ at $20 \mathrm{~K}$ and $2 \mathrm{~T}\left(J_{c}\right.$ $=108000 \mathrm{~A} / \mathrm{cm}^{2}$ ). The transport $J_{c}$ for the $10 \mathrm{wt} \% \mathrm{SiC}-$ doped $\mathrm{MgB}_{2}$ wire is more than an order of magnitude higher than for the best $\mathrm{Fe}$-sheathed $\mathrm{MgB}_{2}$ wire reported to date at $5 \mathrm{~K}$ and $10 \mathrm{~T}$ and $20 \mathrm{~K}$ and $5 \mathrm{~T},{ }^{5,9}$ respectively.

The inset in Fig. 4 shows $T_{c}$ for the nondoped and 10 wt $\%$ doped samples. The $T_{c}$ for the undoped sample is 37.6 $\mathrm{K}$. For the doped samples, $T_{c}$ decreased with increasing doping level. The transition width was typically $0.5 \mathrm{~K}$. It is striking to note that $T_{c}$ has only dropped by $1.5 \mathrm{~K}$ for the 10 wt $\%$ SiC-doped sample. In contrast, $T_{c}$ was depressed by almost $7 \mathrm{~K}$ for $10 \mathrm{wt} \% \mathrm{C}$ substitution for $\mathrm{B}$ in $\mathrm{MgB}_{2} \cdot{ }^{10}$ These results suggest that the higher tolerance of $T_{c}$ to $\mathrm{SiC}$ doping in $\mathrm{MgB}_{2}$ is attributable to the cosubstitution of $\mathrm{B}$ by $\mathrm{C}$ and $\mathrm{Si}$. This is because the atomic radii of $\mathrm{C}(0.077 \mathrm{~nm})$ and $\mathrm{Si}(0.11 \mathrm{~nm})$ atoms are close to that of B $(0.097 \mathrm{~nm})$. Codoping with $\mathrm{SiC}$ counterbalanced the negative effect on $T_{c}$ of the single element doping.

Regarding the mechanism behind the enhancement of $J_{c}$ at higher fields, it is necessary to recognize the special features of $\mathrm{SiC}$ doping. First, in contrast to previous work on doping for improving $J_{c}$, $\mathrm{SiC}$ doping has no densification effect, as evidenced by the fact that the density of doped samples is $1.2 \mathrm{~g} / \mathrm{cm}^{3}$, independent of the doping level. In addition, $\mathrm{SiC}$ doping takes place in the form of substitution and/or addition, ${ }^{8}$ while in the previously reported work, ${ }^{4-6}$ the doping was in the form of additives, not incorporated into crystalline lattice of $\mathrm{MgB}_{2}$.

The transmission electron microscopy images showed a high density of dislocations and a large number of $\sim 10 \mathrm{~nm}$ inclusions inside the grains (Fig. 5). Their concentration increased with the doping level. EDS analysis of the grains revealed the presence of uniformly distributed $\mathrm{Mg}, \mathrm{B}, \mathrm{C}, \mathrm{Si}$, and $\mathrm{O}$ (inset to Fig. 5). This, and the results of XRD, suggests that the inclusion nanoparticles were made of $\mathrm{Mg}_{2} \mathrm{Si}$, or unreacted SiC. All the intragrain defects and the inclusions act as effective pinning centers. Our results suggest that a combination of substitution-induced defects and highly dispersed additives are responsible for the enhanced flux pin-

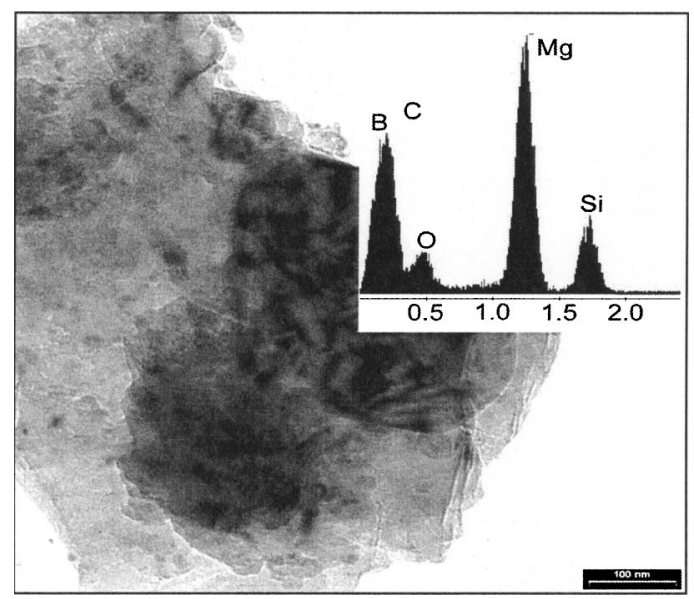

FIG. 5. TEM image showing the intragrain dislocations and nanoparticle inclusions within $\mathrm{MgB}_{2}$ grains. Inset: EDS element analysis of $\mathrm{MgB}_{2}$ grains.

ning. When $\mathrm{SiC}$ reacts with liquid $\mathrm{Mg}$ and amorphous $\mathrm{B}$ at the sintering temperatures, the nanoparticles of $\mathrm{SiC}$ will act as nucleation sites to form $\mathrm{MgB}_{2}$ and other nonsuperconducting phases which can be included within the grains as inclusions. Thus, the reaction-induced products are highly dispersed in the bulk matrix.

Given the ease of production of SiC-doped $\mathrm{MgB}_{2}$, our results significantly strengthen the position of $\mathrm{MgB}_{2}$ as a competitor to more expensive conventional and hightemperature superconductors. It is evident that future $\mathrm{MgB}_{2}$ conductors will be made using a formula of $\mathrm{MgB}_{x} \mathrm{Si}_{y} \mathrm{C}_{z}$ instead of pure $\mathrm{MgB}_{2}$. In summary, we have demonstrated that both the transport and magnetic $J_{c}$, the irreversibility field and the flux pinning of $\mathrm{MgB}_{2}$ are all significantly enhanced through nano-SiC doping, significantly improving the potential of $\mathrm{MgB}_{2}$ for many applications.

The authors thank Dr. T. Silver, Dr. M. J. Qin, Dr. A. Pan, Dr. E. W. Collings, Dr. M. Sumption, and R. Neale for their helpful discussions. This work was supported by the Australian Research Council, Hyper Tech Research Inc., Ohio, and Alphatech International Ltd, NZ.

${ }^{1}$ J. Nagamatsu, N. Nakagawa, T. Muranaka, Y. Zenitani, and J. Akimitsu, Nature (London) 410, 63 (2001).

${ }^{2}$ C. B. Eom, M. K. Lee, J. H. Choi, L. Belenky, X. Song, L. D. Cooley, M. T. Naus, S. Patnaik, J. Jiang, M. Rikel, A. Polyanskii, A. Gurevich, X. Y. Cai, S. D. Bu, S. E. Babcock, E. E. Hellstrom, D. C. Larbalestier, N. Rogado, K. A. Regan, M. A. Hayward, T. He, J. S. Slusky, K. Inumaru, M. K. Haas, and R. J. Cava, Nature (London) 411, 558 (2001).

${ }^{3}$ Y. Bugoslavsky, L. F. Cohen, G. K. Perkins, M. Polichetti, T. J. Tate, R. G. William, and A. D. Caplin, Nature (London) 411, 561 (2001).

${ }^{4}$ Y. Zhao, Y. Feng, C. H. Cheng, L. Zhou, Y. Wu, T. Machi, Y. Fudamoto, N. Koshizuka, and M. Murakami, Appl. Phys. Lett. 79, 1154 (2001).

${ }^{5}$ J. Wang, Y. Bugoslavsky, A. Berenov, L. Cowey, A. D. Caplin, L. F. Cohen, J. L. MacManus Driscoll, L. D. Cooley, X. Song, and D. C. Larbalestier, Appl. Phys. Lett. 81, 2026 (2002).

${ }^{6}$ M. R. Cimberle, M. Novak, P. Manfrinetti, and A. Palenzona, Supercond. Sci. Technol. 15, 34 (2002).

${ }^{7}$ S. X. Dou, X. L. Wang, J. Horvat, D. Milliken, A. H. Li, K. Konstantinov, E. W. Collings, M. D. Sumption, and H. K. Liu, Physica C 361, 79 (2001).

${ }^{8}$ S. X. Dou, A. V. Pan, S. Zhou, M. Ionescu, H. K. Liu, and P. R. Munroe, Supercond. Sci. Technol. (in press).

${ }^{9}$ C. Beneduce, H. L. Suo, P. Toulemonde, N. Musolino, and R. Flukiger, Cond-mat/0203551.

${ }^{10}$ W. Mickelson, J. Cumings, W. Q. Han, and A. Zettl, Phys. Rev. B 65, 052505 (2002). 